\title{
A SHORT PROOF OF THE CLASSICAL EDGE OF THE WEDGE THEOREM
}

\author{
ERIC BEDFORD
}

\begin{abstract}
By solving the $\delta$-equation and using Bochner's theorem on tube domains, one may derive an easy proof of the edge of the wedge theorem in 2 variables.
\end{abstract}

Let $\boldsymbol{R}_{+}^{n}$ and $\boldsymbol{R}_{-}^{n}$ denote the (open) positive and negative cones in $\boldsymbol{R}^{n}$. The classical edge of the wedge theorem states that a function which is analytic on the set $\left\{z \in C^{n}:\left(\operatorname{Re} z_{1}, \cdots, \operatorname{Re} z_{n}\right) \in \boldsymbol{R}_{+}^{n} \cup \boldsymbol{R}_{-}^{n}\right\}$ and continuous where $\left(\operatorname{Re} z_{1}, \cdots, \operatorname{Re} z_{n}\right)=(0, \cdots, 0)$ can be continued holomorphically to the whole space $C^{n}$. We prove a slightly weaker version of this.

THEOREM. Let $W=\left\{z \in C^{2}: \operatorname{Re} z \in R_{+}^{2} \cup R_{-}^{2}\right\}$. If $f \in C^{2}\left(C^{2}\right)$ and if $f$ is analytic on $W$, then $f$ can be continued analytically to $C^{2}$.

Proof. Define two $(0,1)$-forms:

$$
\begin{aligned}
h_{1}(z) & =\bar{\partial} f(z), \quad \operatorname{Re} z_{1}>0, \\
& =0, \quad \text { otherwise; } \\
h_{2}(z) & =\bar{\partial} f(z)-h_{1}(z) .
\end{aligned}
$$

Observe that $\partial f / \partial \bar{z}_{j}$ and $\operatorname{grad}\left(\partial f / \partial \bar{z}_{j}\right)$ vanish on $W$ for $j=1,2$ and therefore on the set $\left\{z: \operatorname{Re} z_{1}=0\right\}$. Thus, the forms $h_{j}$ are continuously differentiable, and $\bar{\partial} h_{j}=0$ for $j=1,2$.

Let $g_{j}$ be functions such that $\bar{\partial} g_{j}=h_{j}$ for $j=1,2$. Then $g_{1}$ is analytic on the set $W_{1}=\left\{\left(z_{1}, z_{2}\right): \operatorname{Re} z_{1}<0\right.$ or $\left.\operatorname{Re} z_{2}>0\right\}$, and it follows from Bochner's theorem on tube domains that there is an entire analytic continuation $G_{1}$ of $g_{1}$. Similarly there is an entire function $G_{2}$ continuing the function $g_{2}$ from the set $\left\{z: \operatorname{Re} z_{1}>0\right.$ or $\left.\operatorname{Re} z_{2}<0\right\}$. Thus $F=f+\left(G_{1}-g_{1}\right)+\left(G_{2}-g_{2}\right)$ is the desired analytic continuation of $f$.

REMARK. The edge of the wedge theorem in $C^{n}(n>2)$ may be deduced from the two-dimensional result along the following lines. If a function satisfies the hypotheses of the above Theorem in $\boldsymbol{C}^{n}$, then it also satisfies them on each complex 2-plane through $(1,1, \cdots, 1)$ and the origin.

Received by the editors March 13, 1973.

AMS (MOS) subject classifications (1970). Primary 32D15.

(C) American Mathematical Society 1974 
By the Theorem for $C^{2}$, the function can be continued analytically to each of these complex 2-planes. The extension defined in this way can be shown to be analytic in $\boldsymbol{C}^{n}$.

\section{REFERENCES}

1. L. Hörmander, An introduction to complex analysis in several variables, Van Nostrand, Princeton, N.J., 1966. MR 34 \#2933.

2. W. Rudin, Lectures on the edge-of-the-wedge theorem, CBMS Regional Conference Series in Math., no. 6, Amer. Math. Soc., Providence, R.I., 1971.

Department of Mathematics, University of Michigan, Ann Arbor, Michigan 48104 